\title{
Uso do Web-OSCE como método de avaliação durante a pandemia de COVID-19
}

\author{
Use of Web-OSCE as an assessment method during the COVID-19 pandemic \\ Uso de Web-OSCE como método de evaluación durante la pandemia COVID-19
}

\section{Resumo}

Objetivo: relatar a experiência do Centro Universitário Christus quanto às modificações na aplicação do Objective Structured Clinical Examination (OSCE) durante a pandemia de COVID-19. Metodologia: relato de experiência de representantes do corpo docente. Resultados: Durante o emergency remote teaching (ERT) na pandemia de COVID-19, os processos avaliativos do módulo Conhecimentos, Habilidades e Atitudes foram adaptados para avaliações tipo WebOSCE, realizados de forma síncrona, com os alunos em seus domicílios, em salas do Google Meet巴. As estações tiveram duração de 5 minutos. No dia da aplicação da prova, os alunos foram confinados em uma sala do Google Meet ${ }^{\circledR}$ para as orientações iniciais. Imediatamente depois, foram divididos em salas virtuais com um fiscal de sala, e orientados a manterem ligados câmera e microfone. O paciente padronizado (PP) foram atores, os quais ficaram responsáveis pela gravação da estação e apresentação do comando ao aluno na tela e no chat. Após a execução da tarefa, o PP encerrou a gravação, e o aluno saiu da sala. O feedback preparado anteriormente foi disponibilizado em vídeo curto ao final da estação. A avaliação das estações foi realizada por análise dos vídeos gravados e pelo preenchimento de checklist, de forma assíncrona. Conclusão: O Web-OSCE mostrou-se um método factível e apresenta potencial para uso em outros cenários, incluindo avaliação de teleconsultas. Esse método pode ser considerado complementar e, junto a outras avaliações remotas do tipo múltipla escolha, pode estimar o ganho cognitivo dos discentes durante o ERT.

Palavras-chave: Avaliação educacional; Educação médica; Educação em saúde; COVID-19; Pandemias; Ensino.

\begin{abstract}
Objective: to report the experience of Centro Universitário Christus regarding changes in the application of the Objective Structured Clinical Examination (OSCE) during COVID-19 pandemic. Methodology: experience report of faculty representatives. Results: During the emergency remote teaching (ERT) in COVID-19 pandemic, the assessment processes of the Knowledge, Skills and Attitudes module were adapted to Web-OSCE type assessments, performed
\end{abstract}


synchronously, with students in their homes, using Google Meet@. The stations lasted for 5 minutes. On application day, students were confined to a Google Meet ${ }^{\circledR}$ room for initial orientation. Immediately afterwards, they were divided into virtual rooms with a room supervisor, and they were instructed to keep the camera and microphone connected. The standardized patient (PP) were actors, who were responsible for recording the station and presenting the command to the student on the screen and in the chat. After the student performed the task, the PP ended the recording, and the student left the room. The previously prepared feedback was made available in a short video at the end of the season. The assessment of the students stations performance was carried out by analyzing the recorded videos and filling out a checklist, asynchronously. Conclusion: The Web-OSCE proved to be a feasible method and it has potential for use in other scenarios, including assessment of teleconsultations. This method can be considered complementary and, together with other remote multiple-choice assessments, can estimate the cognitive gain of students during the ERT.

Keywords: Educational measurement; Medical education; Health education; COVID-19; Pandemics; Teaching.

\section{Resumen}

Objetivo: reportar la experiencia del Centro Universitário Christus sobre cambios en la aplicación del Objective Structured Clinical Examination (OSCE) durante la pandemia de COVID-19. Metodología: relato de experiencia de los representantes de la facultad. Resultados: Durante la enseñanza remota de emergencia (ERT) en la pandemia COVID19, los procesos de evaluación del módulo de Conocimientos, Habilidades y Actitudes se adaptaron a evaluaciones tipo Web-OSCE, realizadas de forma sincrónica, con los estudiantes en sus hogares, utilizando Google Meet ${ }^{\circledR}$. Las estaciones duraron 5 minutos. El día de la solicitud, los estudiantes fueron confinados a una sala de Google Meet ${ }^{\circledR}$ para la orientación inicial. Inmediatamente después, se dividieron en salas virtuales con un supervisor de sala, y se les indicó que mantuvieran la cámara y el micrófono conectados. El paciente estandarizado (PP) fueron los actores, quienes se encargaron de grabar la estación y presentar el comando al alumno en la pantalla y en el chat. Una vez que el alumno realizó la tarea, el PP finalizó la grabación y el alumno abandonó la sala. Los comentarios previamente preparados se pusieron a disposición en un breve video al final de la temporada. La evaluación del desempeño de las estaciones de los estudiantes se llevó a cabo mediante el análisis de los videos grabados y el llenado de una lista de verificación, de forma asincrónica. Conclusión: Web-OSCE demostró ser un método viable y tiene potencial para usarse en otros escenarios, incluida la evaluación de teleconsultas. Este método puede considerarse complementario y, junto con otras evaluaciones remotas de opción múltiple, puede estimar la ganancia cognitiva de los estudiantes durante la ERT.

Palabras clave: Evaluación educacional; Educación médica; Educación en salud; COVID-19; Pandemias; Enseñanza.

\section{Introdução}

No último ano, a partir do momento em que foi decretado estado de pandemia de COVID-19 pela Organização Mundial de Saúde (OMS), observa-se número expressivo de mortes por síndrome respiratória aguda grave, denotando a importância da situação. Até maio de 2021, 162.704.139 casos foram confirmados no mundo, com desfecho fatal em 3.374.052 ocasiões. (World Health Organization [WHO], 2021)

Durante o mesmo período, no Brasil, foram confirmados mais de 15 milhões de casos, com o estado do Ceará apresentando um percentual significativo. Essa situação acarretou a extensão de um decreto estadual publicado em 2020, que promoveu a interrupção de atividades não essenciais ligadas ao comércio, bem como das instituições de ensino públicas e privadas. Como consequência, pela indisponibilidade de atividades presenciais, alguns planos pedagógicos tornaram-se inviáveis, sendo necessário o fornecimento de um sistema norteador com rápida configuração e execução, caracterizando o conceito de emergency remote teaching (ERT). (Ministério da Saúde, 2020; Decreto no 33.519 de 19 de março do Estado do Ceará, 2020; Hodges, Moore, Lockee, Trust, \& Bond, 2020)

A pandemia causou não apenas uma transformação da educação quanto ao modo de ensinar, mas também quanto ao modo de avaliar a aprendizagem, tornando a situação mais complexa com a necessidade de adaptação para aplicação de avaliações de forma remota. (Jaap et al., 2021)

Apesar de haver poucos estudos considerando os desafios da aplicação de avaliações remotas, no ensino médico, tornouse presente não somente a necessidade de adaptação para a aplicação de avaliações de conhecimento por meio de testes de múltipla escolha, mas também a de avaliar habilidades e atitudes frente a problemas pertinentes à prática médica. (Alruwais, Wills, \& Wald, 2018) 
Existem vários métodos possíveis para avaliação prática de estudantes de medicina, incluindo métodos mais tradicionais e antigos - prova oral, short case e long case - criticados pela pouca padronização, confiabilidade e possível viés do avaliador; bem como existem modelos baseados em simulação como o Objective Structured Clinical Examination (OSCE), que garante a segurança do paciente e torna possível a avaliação de três domínios: cognitivo, afetivo e psicomotor. (Majumder et al., 2019)

Outros modelos práticos avaliativos, idealizados inicialmente visando caráter formativo, podem ser citados, tais quais o Mini Clinical Evaluation Exercise (Mini-CEX) e o Direct Observation of Procedural Skills (DOPS), interessantes no contexto de mensurar performance e competência clínica em um ambiente real com feedback imediato ao aluno. Outro método menos comum, em termos de utilização, é o Mini-multiple interview (MMI), desenvolvido pela Universidade de McMaster em 2004, o qual consiste na utilização de estações cronometradas, curtas, de entrevistas envolvendo diferentes competências como criatividade e ética. (Lörwald et al., 2018; Santos, Freire, Barbosa, Figueiredo, \& Costa, 2020)

A pandemia gerou uma disrupção quanto aos modelos vigentes de avaliação, tornando necessária a adoção de ferramentas seguras quanto à trapaça por parte dos alunos, bem como viáveis do ponto de vista tecnológico para docentes e discentes. (Fuller et al., 2020)

São escassos os dados referentes a estratégias avaliativas durante o período pandêmico. Entretanto existem orientações gerais, práticas e relativamente simples, como utilizar o chat para respostas instantâneas a alguma questão, conversão de exame físico para formato digital ou utilização de teleconsultas para essa finalidade. (Said \& Schwartz, 2021)

No Brasil, mais especificamente em um centro universitário de Anápolis-GO, houve modificação quanto à frequência de avaliação dos alunos durante o período remoto, passando de uma avaliação pontual objetiva no formato de múltiplas escolhas para avaliações de cunhos somativos e formativos, semanais, na forma de questionários estruturados, sendo perceptível uma avaliação mais fidedigna do processo ensino-aprendizagem e redução das fraudes, embora tenha sobrecarregado o corpo docente. (Lima et al., 2020)

A partir desse contexto, este artigo tem como objetivo relatar a experiência de um Centro Universitário do nordeste do Brasil quanto às modificações realizadas para a aplicação do OSCE durante o período de pandemia e de distanciamento social.

\section{Metodologia}

A experiência relatada é a de um Centro Universitário pertencente a uma Instituição de Ensino Superior privado, localizado na cidade de Fortaleza, no estado do Ceará. (Pereira et al., 2018)

O Curso de Medicina desta instituição foi iniciado em 2006 e adota, desde o início, um currículo modular integrado e com uso de metodologia híbrida, sendo as atividades de ensino realizadas por meio de Aprendizado Baseado em Problemas (ABP), Aprendizado Baseado em Equipes (ABE) e metodologia tradicional com exposições dialogadas. Quanto às atividades práticas de ensino, estas são realizadas através de simulações com paciente padronizado (atores) no laboratório de habilidades e, durante atividades de vivências clínicas, com pacientes reais e professores em hospitais da rede de atenção à saúde do Estado e do Município e em ambulatórios da clínica escola de saúde da instituição. Ambas as atividades são realizadas por alunos do primeiro ao oitavo semestre.

Quanto aos métodos de avaliação, estes são realizados ao longo do semestre. Avaliações formativas são realizadas durante as atividades práticas de ensino e durante as sessões de ABP. Quanto a avaliações somativas, estas são realizadas por meio de testes com questões de múltipla escolha e provas práticas do tipo OSCE no laboratório de habilidades com paciente padronizado (atores).

Durante o período de distanciamento social, diversas metodologias de ensino e avaliação precisaram de adaptações, principalmente avaliações tipo OSCE. 


\section{Resultados}

Neste período de isolamento social, a instituição necessitou inovar na realização das atividades do módulo de Conhecimentos, Habilidades e Atitudes (CHA), as quais eram previamente realizadas no ambulatório de habilidades. Os alunos passaram a ter encontros à distância, síncronos, em pequenos grupos de dez estudantes, utilizando salas da plataforma Google Meet®, com o professor e um paciente padronizado (ator). Durante essas atividades didáticas, foi possível trabalhar conhecimentos, habilidades de comunicação e atitudes, por meio da interação dos alunos com o paciente padronizado, com a realização sequencial de debriefing. Entretanto, devido a pandemia, as habilidades procedurais ficaram de serem repostas em um segundo momento, após a liberação pelas autoridades sanitárias do estado.

Quanto aos métodos de avaliação do módulo CHA, foi possível realizar avaliação formativa dos alunos, ao final de cada atividade remota síncrona. Porém, ficou o desafio da realização de avaliação do tipo OSCE ao final de cada período.

Diante desse desafio, foi proposta a realização de avaliação do tipo OSCE, porém utilizando a rede de internet de forma síncrona, com acesso do aluno do próprio domicílio, em salas virtuais do Google Meet@. O paciente padronizado seria um ator, e a supervisão seria feita pelos professores. Utilizou-se a denominação de Web-OSCE, previamente descrita em alguns trabalhos na literatura. (Novack et al., 2002)

Durante a preparação, os professores receberam um encarte digital via e-mail com toda a orientação sobre como elaborar as estações. Todos os alunos também receberam um encarte digital via e-mail contendo as orientações sobre os passos da avaliação. As orientações para o preparo foram inicialmente sobre o que seria possível avaliar por meio desse método. As estações deveriam ter como foco a avaliação de conhecimentos, de habilidades de comunicação e de atitudes frente a uma situação clínica previamente trabalhada nas aulas do módulo CHA.

Os professores de cada período do módulo CHA foram orientados a preparar duas estações para avaliação do tipo OSCE, com duração de 5 minutos e com comando claro e objetivo. Foram solicitados também materiais que o paciente padronizado precisasse apresentar ao aluno (exames, receitas, foto de lesão, etc.) e o checklist de cada estação elaborado de forma que cada item fosse avaliado como: não realizou, realizou parcialmente ou realizou completamente. Além disso, os professores prepararam o feedback de cada estação, gravado em vídeo curto, comentando sobre as habilidades trabalhadas e as ações necessárias para o êxito diante daquela situação.

No dia da aplicação do teste, a preparação dos atores foi realizada pelo professor responsável pela elaboração das estações. A orientação quanto ao script foi realizada presencialmente em uma sala ampla, mantendo o distanciamento de 2 metros, com uso de máscara e de álcool em gel para higienização das mãos.

Todos os alunos foram confinados em uma sala virtual do Google Meet®, a qual o professor responsável pela elaboração da estação acessou e passou as orientações iniciais. Após isso, os estudantes foram divididos em salas virtuais para pequenos grupos com 10 a 20 alunos, junto a um fiscal de sala, e orientados a ficarem com câmera e microfone ligados.

Quanto às orientações adicionais aos alunos, foram orientados a ficarem em ambiente bem iluminado, sem interferências e sem a presença de outras pessoas, além do próprio aluno. Foram alertados que o fiscal poderia entrar em contato com o aluno a qualquer momento durante a avaliação, pedindo-lhe que mostrasse o ambiente no qual estava sendo realizada a sua prova. Foi solicitado ainda que o estudante não se ausentasse do local de prova, nem desligasse a câmera, mantendo seu equipamento carregado e o carregador no local de prova. Não foi permitido o uso de planos de fundo nas salas virtuais, e o aluno foi orientado a manter a câmera apontada para o rosto durante toda a prova.

Foi permitido o uso de uma folha rascunho, desde que esta fosse mostrada (frente e verso) antes do início da estação. Os alunos foram orientados a usarem vestimenta adequada e jaleco, não sendo permitido o uso de artigos de chapelaria como bonés, chapéus, viseiras, gorro ou similares. Também não foi permitido o uso de fones de ouvido, headphones ou protetores auriculares. 
Durante a estação, assim que o aluno entrou na sala, o paciente padronizado iniciou a gravação da estação, então o aluno se identificou para a câmera. Em seguida, o comando da estação foi apresentado ao aluno, que após lê-lo iniciou a tarefa interagindo com o paciente padronizado. Durante toda a estação, o comando da estação ficou disponibilizado também no chat, podendo ser consultado pelo aluno durante a realização da tarefa. Após o término da estação, o paciente padronizado finalizou a gravação da sala virtual e o aluno saiu da sala.

A avaliação das estações foi realizada pelo grupo de professores do módulo CHA, por meio da análise dos vídeos gravados e do preenchimento de checklist, após o término da prova, de forma assíncrona (em outro dia e horário). Já em relação ao feedback de cada estação, não foi possível realizá-lo de forma individualizada, sendo disponibilizado em vídeo após o término da prova para toda a turma.

Os passos para a realização do Web-OSCE estão ilustrados na Figura 1.

Figura 1. Passos realizados durante a avaliação do tipo Web-OSCE.

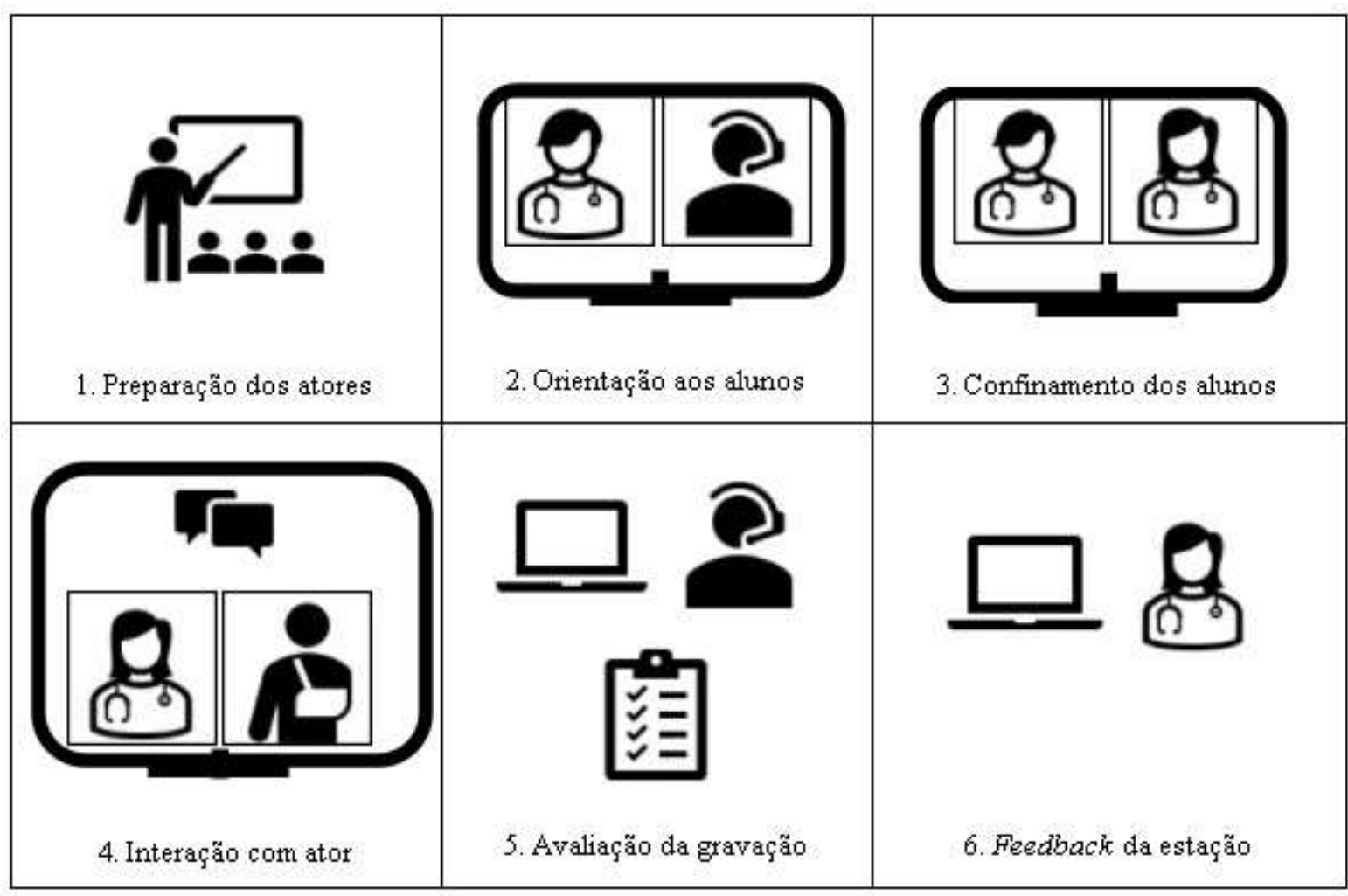

Fonte: Figura elaborada pelos autores, com o uso dos ícones disponíveis gratuitamente pelo programa Microsoft Office®.

\section{Discussão}

O Web-OSCE, ferramenta de avaliação inovadora e necessária para a atual situação, mostra-se como um meio flexível e conveniente, gerando várias experiências a serem refletidas. É válido ressaltar que o método avaliativo descrito permite a continuidade de uma avaliação padronizada quanto à capacidade clínica do aluno, semelhante ao modelo presencial. O OSCE tradicional é uma valiosa ferramenta para a avaliação das competências clínicas e atitudinais, sendo considerado o método de escolha para avaliação das habilidades procedurais. O Web-OSCE, por sua vez, mostra-se especialmente útil para a avaliação das habilidades clínicas necessárias para a prática de teleconsultas, modalidade que apresenta tendência crescente devido ao incremento dos serviços de telemedicina desde o início da pandemia. (Khamisa, Halman, Desjardins, Jean, \& Pugh, 2018; Palmer et al., 2015) 
Uma outra possibilidade é a sua utilização para a avaliação de competências clínicas que não envolvam habilidades procedurais, em locais com limitação de infraestrutura física para a execução de um OSCE tradicional, como método avaliativo complementar. Além disso, o Web-OSCE pode ser uma estratégia para a avaliação das habilidades de comunicação e atitudinais em cursos de especialização ou de aperfeiçoamento realizados à distância.

Os alunos da instituição, em uma pesquisa de satisfação aplicada a todos os semestres que participaram do Web-OSCE, consideraram o modo avaliativo como sendo, de uma maneira geral, bem administrado, estruturado e condizente com o conteúdo ministrado nas aulas remotas, embora relativamente estressante. Esse perfil de resultado é semelhante a outros dados de literatura sobre o OSCE presencial, no qual mais de $75 \%$ dos estudantes entrevistados consideraram alto grau de ansiedade durante a avaliação, embora o método tenha sido considerado aplicável, abrangente, justo e transparente por estes alunos. (Jaap et al., 2021)

Para que a realização do Web-OSCE seja satisfatória, é necessária uma série de fatores: equipe de suporte em tecnologia da informática, plano operacional, treinamento dos atores, inclusive para a utilização das ferramentas tecnológicas, acesso de internet adequado para todos os participantes, dispositivos com áudios/vídeos, sendo extremamente relevante o feedback de todos os envolvidos. Além de toda a implementação técnica, a validade das questões aplicadas é de extrema relevância, com adequado grau de dificuldade e compatível com o conteúdo pedagógico. (Major, Sawan, Vognsen, \& Jabre, 2020; Hytönen et al., 2020)

Há algumas limitações nesse relato de experiência, visto que não existem dados suficientes em literatura referentes ao escore final dos alunos, bem como à consequência na formação dos egressos. Tivemos algumas ocorrências pontuais indesejadas, como o atraso de professores para o momento da avaliação e a indisponibilidade imediata de profissionais da informática. Isso pode inclusive inviabilizar o processo avaliativo para algumas instituições.

Um aspecto limitante do Web-OSCE é a maior dificuldade em avaliar algumas competências clínicas, tais quais interação e destreza. Outra desvantagem do OSCE virtual é a impossibilidade de se mensurar habilidades técnicas, o que poderia ser minimizado com sistemas mais interativos por meio de aprimoramento audiovisual. (Khamisa et al., 2018; Hytönen et al., 2020; Martinho \& Griffin, 2020)

Apesar das restrições impostas pela pandemia, é fundamental a continuidade de avaliações, minimizando o quanto possível a exposição ao vírus da COVID-19. Nesse contexto, o Web-OSCE permite a adoção de um sistema flexível, minimizando o tempo de deslocamento tanto para docentes e discentes, assegurando a avaliação de algumas competências, como a comunicação, a qualquer momento e local, apoiado na ideia de que experiências virtuais ocorrerão com frequência na prática clínica por meio da telemedicina. A ferramenta discutida é considerada complementar e, junto a outras avaliações remotas do tipo múltipla escolha, pode estimar o ganho cognitivo e de algumas habilidades dos discentes. (Miao, 2021; Looi et al., 2021)

\section{Conclusão}

A manutenção das atividades letivas e dos processos avaliativos nos cursos da área da saúde durante o período de distanciamento social foi um grande desafio, colocando em prova a resiliência institucional das entidades de ensino médico. A implementação de avaliações remotas seguras foi necessária, e para isso utilizaram-se estratégias previamente descritas na literatura, como o Web-OSCE.

Nesse sentido, esse método se mostrou factível, podendo ser utilizado de forma complementar, para avaliar as competências clínicas dos alunos durante o ERT. Adicionalmente, com o incremento das modalidades de telemedicina, o $\mathrm{Web}$ OSCE poderá se consolidar como modo avaliativo para as práticas de teleconsultas. Estudos futuros, referentes ao impacto desse método nos escores finais dos alunos e na formação dos egressos, serão importantes para guiar a sua implementação em diferentes contextos. 


\section{Referências}

Alruwais, N., Wills, G., \& Wald, M. (2018). Advantages and challenges of using e-assessment. International Journal of Information and Education Technology, 8(1), 34-37.

Brasil. (2020). Painel Coronavírus. Ministério da Saúde, Secretaria de Vigilância em Saúde.

Ceará. (2020). Decreto no 33.519 de 19 de março de 2020. Intensifica as medidas para o enfrentamento da infecção humana pelo novo coronavírus. Diário Oficial do Estado, 56, 1-2

Fuller, R., Joynes, V., Cooper, J., Boursicot, K., \& Roberts, T. (2020). Could COVID-19 be our 'There is no alternative' (TINA) opportunity to enhance assessment? Medical Teacher, 42(7),781-786.

Hodges, C., Moore, S., Lockee, B., Trust, T., \& Bond, A. (2020). The difference between emergency remote teaching and online learning. Educause Review.

Hytönen, H., Näpänkangas, R., Karaharju-Suvanto, T., Eväsoja, T., Kallio, A., Kokkari, A., \& Lahti, S. (2020). Modification of national OSCE due to COVID19: Implementation and students' feedback. European Journal of Dental Education, 00, 1-10.

Jaap, A., Dewar, A., Duncan, C., Fairhurst, K., Hope, D., \& Kluth, D. (2021). Effect of remote online exam delivery on student experience and performance in applied knowledge tests. BMC Medical Education, 21(1), 86.

Khamisa, K., Halman, S., Desjardins, I., Jean, M. S., \& Pugh, D. (2018). The implementation and evaluation of an e-learning training module for objective structured clinical examination raters in Canada. Journal of Educational Evaluation for Health Professions, 15, 18.

Lima, M. M., Oliveira, A. M., Araújo Freitas, A., Lima, C. S., Bernardes, C. T. V., Sugita, D. M., \& Arruda, J. T. (2020). Estratégias avaliativas no ensino remoto: Avaliação terminal ou contínua? Anais do Seminário de Atualização de Práticas Docentes, 2(2), $126-132$.

Looi, J. C. L., Maguire, P., Bonner, D., Reay, R. E., Finlay, A. J. F., Keightley, P., \& Kramer, D. (2021). Conduct and evaluation of final-year medical student summative assessments in psychiatry and addiction medicine during COVID-19: An Australian University Medical School experience. Australasian Psychiatry: Bulletin of Royal Australian and New Zealand College of Psychiatrists, 00, 1-5.

Lörwald, A. C., Lahner, F. M., Nouns, Z. M., Berendonk, C., Norcini, J., Greif, R., \& Huwendiek, S. (2018). The educational impact of Mini-Clinical Evaluation Exercise (Mini-CEX) and Direct Observation of Procedural Skills (DOPS) and its association with implementation: A systematic review and meta-analysis. PloS one, 13(6), e0198009.

Major, S., Sawan, L., Vognsen, J., \& Jabre, M. (2020). COVID-19 pandemic prompts the development of a Web-OSCE using Zoom teleconferencing to resume medical students' clinical skills training at Weill Cornell Medicine-Qatar. BMJ Simulation \& Technology Enhanced Learning, 0, 1-2.

Majumder, M. A. A., Kumar, A., Krishnamurthy, K., Ojeh, N., Adams, O. P., \& Sa, B. (2019). An evaluative study of objective structured clinical examination (OSCE): students and examiners perspectives. Advances in Medical Education and Practice, 10, $387-397$.

Martinho, F. C., \& Griffin, I. L. (2020). OSCE online teaching in predoctoral endodontics. Journal of Dental Education, 10.1002/jdd.12418. Advance online publication.

Miao, J. H. (2021). Adapting medical education initiatives through team-based e-learning, telemedicine objective structured clinical exams, and student-led community outreach during the COVID-19 pandemic. JMIR Medical Education, 7(2), e26797.

Novack, D. H., Cohen, D., Peitzman, S. J., Beadenkopf, S., Gracely, E., \& Morris, J. (2002). A pilot test of WebOSCE: A system for assessing trainees' clinical skills via teleconference. Medical Teacher, 24(5), 483-487.

Palmer, R. T., Biagioli, F. E., Mujcic, J., Schneider, B. N., Spires, L., \& Dodson, L. G. (2015). The feasibility and acceptability of administering a telemedicine objective structured clinical exam as a solution for providing equivalent education to remote and rural learners. Rural and Remote Health, 15 (4), 3399.

Pereira, A. S., Shitsuka, D. M., Parreira, F. J., \& Shitsuka, R. (2018). Metodologia da Pesquisa Científica. (1. ed.) Rio Grande do Sul: UAB/NTE/UFSM. Disponível em https://www.ufsm.br/app/uploads/sites/358/2019/02/Metodologia-da-Pesquisa-Cientifica_final.pdf

Santos, S., Freire, C., Barbosa, I., Figueiredo, H., \& Costa, M. J. (2020). Assessing transversal competencies for the future of graduate work: An adaptation of the multiple mini-interviews method. Proceedings of ICERI2020 Conference, 4112-4122.

Said, J. T., \& Schwartz, A. W. (2021). Remote medical education: Adapting kern's curriculum design to tele-teaching. Medical Science Educator, 31, 805-812. World Health Organization [WHO]. 2021. Weekly Operational Update on COVID-19, 55, 1-5. 